\title{
Can we identify meaningful epigenetic effects on human brain function and related risk for mental illness?
}

\begin{abstract}
"...a handful of recent studies have successfully begun to combine epigenetics and in vivo brain imaging techniques, such as functional $\mathrm{MRI}$, to examine associations between peripheral markers of DNA cytosine methylation and clinically relevant brain function."
\end{abstract}

First draft submitted: 5 August 2016; Accepted for publication: 8 August 2016; Published online: 7 September 2016

Keywords: DNA methylation $\bullet$ environmental stress $\bullet$ human brain function $\bullet$ mental illness

Research on epigenetic modifiers of human brain function and related risk for mental illness faces the unique and seemingly insurmountable challenge of studying tissue that is simultaneously one of the most functionally complex and least accessible in the human body. Nevertheless, a handful of recent studies have successfully begun to combine epigenetics and in vivo brain imaging techniques, such as functional MRI, to examine associations between peripheral markers of DNA cytosine methylation and clinically relevant brain function [1]. Alongside these promising beginnings, a more long-standing literature has demonstrated that environmental stress, particularly early in life, can alter the epigenetic landscape to increase risk for a variety of disorders, including mental illness [2-4]. Taken together, these parallel lines of work show significant promise for bridging the link from environmental risk to mechanisms of psychopathology and possible therapeutic targets. However, many open questions and limitations have prevented further synergy and more rapid advances. Here we briefly consider these challenges and discuss potential avenues for moving forward through the lens of our ongoing work.

The field of imaging epigenetics has first and foremost struggled with the methodological and conceptual challenge of establishing meaningful links between DNA methylation patterns in peripheral tissue and those present in the brain. Indeed, the methylome, like the rest of the epigenome, is expected not only to vary from cell type to cell type, but also to be part of the very mechanism that determines the fate of each cell. Nonetheless, more and more studies suggest a broad, though not complete, convergence between methylation patterns in brain and those in blood or saliva [5-7]. This convergence reinforces the possibility that peripheral measures of DNA methylation may serve as informative, albeit crude, indices of the brain's current epigenetic state. Importantly, however, all studies that have demonstrated cross-tissue methylome convergence have also invariably shown that the strength of this convergence is likely locus- and gene-specific, with some genes or genomic regions showing more cross-tissue similarity than others. Uncovering the functional impact of methylation patterns on gene expression levels within and across different tissues presents a further challenge that may similarly have to be tackled on a geneor genomic element-specific basis [8]. Thus, it comes as no surprise that imaging epigenetic studies so far have focused primarily on well-characterized genes and genomic loci [1].

Perhaps the most studied gene in psychiatric and imaging genetics over the past two decades is SLC6A4, which encodes the human serotonin transporter (SERT). A common sequence variant in the distal promoter of SLC6A4 has been associated

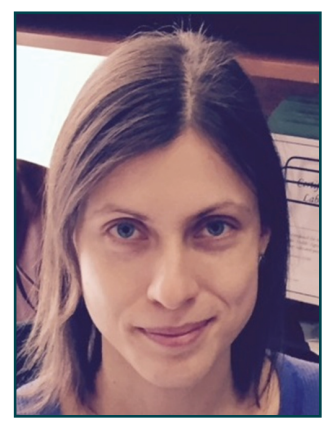

Yuliya S Nikolova

Author for correspondence: Campbell Family Mental Health Research Institute, Centre for Addiction \& Mental Health, 250 College Street, Toronto, ON, M5T 1R8, Canada yuliya.nikolova@camh.ca

Johnna R Swartz Department of Human Ecology, University of California, Davis, CA 95616, USA

\section{Ahmad R Hariri}

Laboratory of

NeuroGenetics,

Department of Psychology

\& Neuroscience, Duke

University, Durham, NC 27708, USA 
with reduced gene expression [9], increased threatrelated brain function [10,11] and heightened risk for depression [12,13]. Importantly, the latter finding only emerges consistently in the context of early or recent life stress, suggesting this risk pathway may be epigenetically regulated. Drawing on these early findings, in our recent work, we showed that average methylation percentage across $20 \mathrm{CpG}$ sites in the proximal promoter of SLC6A4, measured in peripheral blood or saliva, is positively correlated with amygdala reactivity to threat [14], a phenotype independently implicated in risk for depression and anxiety [15] and consistent with reduced SERT function [16]. This association was present in two independent samples representing different developmental stages - adolescence and young adulthood - and was robust to the specific peripheral tissue sampled. Furthermore, we found that methylation in the same genomic region was negatively associated with SERT mRNA expression in human postmortem amygdala tissue, providing preliminary insight into the molecular mechanisms that may underlie the observed association with brain function.

“...we found that an increase in SLC6A4 methylation measured in blood over the 2-year period was positively associated with an increase in amygdala function."

Since our original study, others have shown that SLC6A4 promoter methylation is associated with increased activity in multiple regions that are structurally and functionally interconnected with the amygdala [17] as well as increased intrinsic functional connectivity of the amygdala and these same regions [18]. In parallel, an accumulating body of research suggests SLC6A4 promoter methylation is higher in individuals exposed to stress early in life [19-22], providing valuable clues as to the potential origins of the observed methylation patterns. Yet, none of these studies have examined the temporal trajectories of these patterns in the same individuals, or integrated measures of brain function, environmental adversity and psychopathology risk within the same longitudinal framework.

Building on these studies and our work examining cross-sectional associations between peripheral SLC6A4 promoter methylation and amygdala function [14], we recently examined these associations longitudinally. We did so by evaluating whether change in peripheral SLC6A4 methylation across 2 years was associated with change in amygdala function during the same period in a sample of adolescents assessed cross-sectionally in our earlier study [14]. Notably and consistent with our earlier findings, we found that an increase in SLC6A4 methylation measured in blood over the 2-year period was positively associated with an increase in amygdala function [23]. This finding lends further credibility to the original association we observed and suggests DNA methylation in peripheral tissue may not only help us index aspects of the brain's current functional state, but also serve as a relatively inexpensive and easy-to-assess peripheral marker of changes occurring at the level of the brain over time.

We also addressed two other questions in this study: were there environmental risk factors that predicted this change in SLC6A4 methylation and what were the consequences of these increases in methylation and amygdala function for future depression symptoms? In answer to the first question, we found that, out of several risk factors examined, the only significant predictor of increases in SLC6A4 methylation over time was low socioeconomic status (SES). Thus, we found that adolescents growing up in lower SES households evidenced greater increases in SLC6A4 methylation over time, and indirectly, greater increases in amygdala reactivity to threat. In answer to the second question, we found that greater increases in amygdala reactivity predicted greater increases in depression symptoms 1 year later, specifically for adolescents who had a positive family history of depression. Overall, then, we observed an indirect effect wherein lower SES predicted greater increases in SLC6A4 methylation, which predicted greater increases in amygdala reactivity, which, in turn, predicted greater increases in depression symptoms.

These preliminary results, though promising, highlight several critical limitations to consider moving forward. One notable limitation is the relatively small effect sizes for each of the paths examined. For instance, SES only explained $5 \%$ of the variance in SLC6A4 methylation, and SLC6A4 methylation only explained $8 \%$ of the variance in amygdala function. It is therefore very likely the case that we will need a large number of such biomarkers before we are able to explain clinically relevant amounts of variance in brain function and associated symptoms. Likewise, the changes in methylation we observed over time were very small - on the order of $2-3 \%$. It is unclear, currently, how these small changes in methylation may affect gene expression at a level that would affect brain function and symptomatology or whether they conform to the same cross-tissue convergence trends observed for large-scale methylation patterns.

Our work also raises important questions as to the timing and origin of the observed changes in methylation. We examined changes in SLC6A4 methylation over a 2-year window, but it is quite possible that changes might occur on a much shorter time scale. Identifying the timing of these changes and the time scales over which they occur will be important in 
thinking about their potential use as biomarkers of risk. A related question, and a critical future direction for this work, is to examine the origin of these epigenetic patterns and their trajectories over a longer period of time. One study suggests that behaviorally relevant SLC6A4 promoter methylation changes in response to stress can begin prenatally [24] or as early as the first days after birth [25,26]. Additionally, transgenerational transmission may also play a role in shaping these patterns [27], raising the possibility that it is not only the early individual but also the parental environment that contributes to pathways of risk. Another consideration for this exciting line of work lies in the steady methylation changes that occur over the course of a lifetime [28] starting from birth [29,30]. As stress and poverty are known to contribute to accelerated epigenetic aging [31,32], a useful question to explore is whether environmental stress may increase risk for mental illness at least in part by interfering with (i.e., inappropriately accelerating, decelerating or reversing) normal age-dependent trajectories of epigenetic change.

Although prospective longitudinal imaging epigenetic studies will be uniquely poised to identify trajectories of risk, their observed associations will remain correlational. To develop mechanistic insight into the origin of any correlational effects, the imaging epigenetics field needs to join forces with preclinical molecular biology and neuroscience, where brain,

\section{References}

1 Nikolova YS, Hariri AR. Can we observe epigenetic effects on human brain function? Trends Cogn. Sci. 19(7), 366-373 (2015).

2 Booij L, Wang D, Levesque ML, Tremblay RE, Szyf M. Looking beyond the DNA sequence: the relevance of DNA methylation processes for the stress-diathesis model of depression. Philos. Trans. R. Soc. Lond. B. Biol. Sci. 368(1615), 20120251 (2013).

3 Klengel T, Binder EB. Epigenetics of stress-related psychiatric disorders and gene $\times$ environment interactions. Neuron 86(6), 1343-1357 (2015).

4 Meaney MJ, Szyf M. Environmental programming of stress responses through DNA methylation: life at the interface between a dynamic environment and a fixed genome. Dialogues Clin. Neurosci. 7(2), 103-123 (2005).

5 Tylee DS, Kawaguchi DM, Glatt SJ. On the outside, looking in: a review and evaluation of the comparability of blood and brain '-omes'. Am. J. Med. Genet. B Neuropsychiatr. Genet. 162B(7), 595-603 (2013).

6 Byun HM, Siegmund KD, Pan F et al. Epigenetic profiling of somatic tissues from human autopsy specimens identifies tissue- and individual-specific DNA methylation patterns. Hum. Mol. Genet. 18(24), 4808-4817 (2009). behavior, environment, DNA methylation and other aspects of the epigenome can be manipulated independently to establish causal relationships. Although a great deal is already known about the molecular mechanisms of stress-related DNA methylation change [3], targeted investigations in animal models, conducted in close parallel with more extensive and possibly intervention-focused longitudinal imaging epigenetic studies in humans, are likely to yield better translational insight. Furthermore, rapid translation between these complementary fields will likely offer the best hope for not only establishing the contribution of the functional epigenome to risk for mental illness with greater certainty, but also identifying ways to reverse or prevent illness altogether.

\section{Financial \& competing interests disclosure}

YS Nikolova is supported by a Banting Postdoctoral Fellowship from the Canadian Institutes of Health Research (CIHR). JR Swartz is supported by the Center for the Study of Adolescent Risk and Resilience (P30DA023026) and through NIH grant R01AG049789. AR Hariri is supported by NIH grants R01DA033369 and R01AG049789. The authors have no other relevant affiliations or financial involvement with any organization or entity with a financial interest in or financial conflict with the subject matter or materials discussed in the manuscript apart from those disclosed.

No writing assistance was utilized in the production of this manuscript.

7 Smith AK, Kilaru V, Klengel T et al. DNA extracted from saliva for methylation studies of psychiatric traits: evidence tissue specificity and relatedness to brain. Am. J. Med. Genet. B Neuropsychiatr. Genet. 168B(1), 36-44 (2015).

8 Davies MN, Volta M, Pidsley R et al. Functional annotation of the human brain methylome identifies tissue-specific epigenetic variation across brain and blood. Genome Biol. 13(6), R43 (2012).

9 Lesch KP, Bengel D, Heils A et al. Association of anxietyrelated traits with a polymorphism in the serotonin transporter gene regulatory region. Science 274(5292), 1527-1531 (1996).

10 Hariri AR, Mattay VS, Tessitore A et al. Serotonin transporter genetic variation and the response of the human amygdala. Science 297(5580), 400-403 (2002).

11 Murphy SE, Norbury R, Godlewska BR et al. The effect of the serotonin transporter polymorphism (5-HTTLPR) on amygdala function: a meta-analysis. Mol. Psychiatry 18(4), 512-520 (2013).

12 Caspi A, Sugden K, Moffitt TE et al. Influence of life stress on depression: moderation by a polymorphism in the 5-HTT gene. Science 301(5631), 386-389 (2003).

13 Karg K, Burmeister M, Shedden K, Sen S. The serotonin transporter promoter variant $(5-H T T L P R)$, stress, and 
depression meta-analysis revisited: evidence of genetic moderation. Arch. Gen. Psychiatry 68(5), 444-454 (2011).

14 Nikolova YS, Koenen KC, Galea S et al. Beyond genotype: serotonin transporter epigenetic modification predicts human brain function. Nat. Neurosci. 17(9), 1153-1155 (2014).

15 Swartz JR, Knodt AR, Radtke SR, Hariri AR. A neural biomarker of psychological vulnerability to future life stress. Neuron 85(3), 505-511 (2015).

16 Bigos KL, Pollock BG, Aizenstein HJ, Fisher PM, Bies RR, Hariri AR. Acute 5-HT reuptake blockade potentiates human amygdala reactivity. Neuropsychopharmacology 33(13), 3221-3225 (2008).

17 Frodl T, Szyf M, Carballedo A et al. DNA methylation of the serotonin transporter gene (SLC6A4) is associated with brain function involved in processing emotional stimuli. J. Psychiatry Neurosci. 40 (2), 140180 (2015).

18 Muehlhan M, Kirschbaum C, Wittchen HU, Alexander N. Epigenetic variation in the serotonin transporter gene predicts resting state functional connectivity strength within the salience-network. Hum. Brain Mapp. 36(11), 4361-4371 (2015).

19 Kang HJ, Kim JM, Stewart R et al. Association of SLC6A4 methylation with early adversity, characteristics and outcomes in depression. Prog. Neuropsychopharmacol. Biol. Psychiatry 44C, 23-28 (2013).

20 Ouellet-Morin I, Wong CC, Danese A et al. Increased serotonin transporter gene (SERT) DNA methylation is associated with bullying victimization and blunted cortisol response to stress in childhood: a longitudinal study of discordant monozygotic twins. Psychol. Med. 43(9), 1813-1823 (2013).

21 Beach SR, Brody GH, Todorov AA, Gunter TD, Philibert RA. Methylation at SLC6A4 is linked to family history of child abuse: an examination of the Iowa Adoptee sample. $A m$. J. Med. Genet. B Neuropsychiatr. Genet. 153B(2), 710-713 (2010).

22 Duman EA, Canli T. Influence of life stress, 5-HTTLPR genotype, and SLC6A4 methylation on gene expression and stress response in healthy Caucasian males. Biol. Mood Anxiety Disord. 5, 2 (2015).

23 Swartz JR, Hariri AR, Williamson DE. An epigenetic mechanism links socioeconomic status to changes in depression-related brain function in high-risk adolescents. Mol. Psychiatry doi:10.1038/mp.2016.82 (2016) (Epub ahead of print).

24 Devlin AM, Brain U, Austin J, Oberlander TF. Prenatal exposure to maternal depressed mood and the MTHFR C677T variant affect SLC6A4 methylation in infants at birth. PLoS ONE 5(8), e12201 (2010).

25 Montirosso R, Provenzi L, Giorda R et al. SLC6A4 promoter region methylation and socio-emotional stress response in very preterm and full-term infants. Epigenomics 8(7), 895-907 (2016).

26 Montirosso R, Provenzi L, Fumagalli M et al. Serotonin transporter gene (SLC6A4) methylation associates with neonatal intensive care unit stay and 3-month-old temperament in preterm infants. Child Dev. 87(1), 38-48 (2016).

27 Szyf M. Nongenetic inheritance and transgenerational epigenetics. Trends Mol. Med. 21(2), 134-144 (2015).

28 Fraga MF, Ballestar E, Paz MF et al. Epigenetic differences arise during the lifetime of monozygotic twins. Proc. Natl Acad. Sci. USA 102(30), 10604-10609 (2005).

29 Levesque ML, Casey KF, Szyf M et al. Genome-wide DNA methylation variability in adolescent monozygotic twins followed since birth. Epigenetics 9(10), 1410-1421 (2014).

30 Horvath S. DNA methylation age of human tissues and cell types. Genome Biol. 14(10), R115 (2013).

31 Zannas AS, Arloth J, Carrillo-Roa $\mathrm{T}$ et al. Lifetime stress accelerates epigenetic aging in an urban, African-American cohort: relevance of glucocorticoid signaling. Genome Biol. 16, 266 (2015)

32 Simons RL, Lei MK, Beach SR et al. Economic hardship and biological weathering: the epigenetics of aging in a US sample of black women. Soc. Sci. Med. 150, 192-200 (2016). 